\title{
Global Existence and Decay for a System of Two Singular Nonlinear Viscoelastic Equations with General Source and Localized Frictional Damping Terms
}

\author{
Salah Mahmoud Boulaaras (D), ${ }^{1,2}$ Rafik Guefaifia, ${ }^{3}$ Nadia Mezouar, ${ }^{4}$ \\ and Ahmad Mohammed Alghamdi ${ }^{5}$ \\ ${ }^{1}$ Department of Mathematics, College of Sciences and Arts, Al-Rass, Qassim University, Saudi Arabia \\ ${ }^{2}$ Laboratory of Fundamental and Applied Mathematics of Oran (LMFAO), University of Oran 1, Ahmed Benbella., Algeria \\ ${ }^{3}$ Department of Mathematics, College of Exact Sciences, University of Tebessa, Tebessa 12002, Algeria \\ ${ }^{4}$ Mascara University, Faculty of Economics, Mascara, Algeria \\ ${ }^{5}$ Department of Mathematical Sciences, College of Applied Science, Umm Al-Qura University, Saudi Arabia
}

Correspondence should be addressed to Salah Mahmoud Boulaaras; s.boularas@qu.edu.sa

Received 19 July 2020; Revised 21 August 2020; Accepted 29 August 2020; Published 9 September 2020

Academic Editor: Fanglei Wang

Copyright (c) 2020 Salah Mahmoud Boulaaras et al. This is an open access article distributed under the Creative Commons Attribution License, which permits unrestricted use, distribution, and reproduction in any medium, provided the original work is properly cited.

The current paper deals with the proof of a global solution of a viscoelasticity singular one-dimensional system with localized frictional damping and general source terms, taking into consideration nonlocal boundary condition. Moreover, similar to that in Boulaaras' recent studies by constructing a Lyapunov functional and use it together with the perturbed energy method in order to prove a general decay result.

\section{Introduction}

The evolution problem with integral conditions is related with many branches of sciences ([1-6]). Cause of this, interest in it occurs naturally in inflation cosmology, nuclear physics, supersymmetric field theories, and quantum mechanics (see for example [2,7]). Later, by the motivation of this work, some authors gave necessary and sufficient conditions for the hyperbolic equation with source term (see, e.g., [8-10]).

This manuscript is devoted to the study of the global existence and decay for a system of two singular one-dimensional nonlinear viscoelastic equations with general source terms 


$$
\left\{\begin{array}{l}
u_{t t}-\frac{1}{x}\left(x u_{x}\right)_{x}+\int_{0}^{t} g_{1}(t-s) \frac{1}{x}\left(x u_{x}(x, s)\right)_{x} d s+\mu(x) u_{t}=f_{1}(u, v), \text { in }(0, L) \times(0, T), \\
v_{t t}-\frac{1}{x}\left(x v_{x}\right)_{x}+\int_{0}^{t} g_{2}(t-s) \frac{1}{x}\left(x v_{x}(x, s)\right)_{x} d s+\mu(x) v_{t}=f_{2}(u, v), \text { in }(0, L) \times(0, T), \\
u(x, 0)=u_{0}(x), u_{t}(x, 0)=u_{1}(x), x \in(0, L), \\
v(x, 0)=v_{0}(x), v_{t}(x, 0)=v_{1}(x), x \in(0, L), \\
u(L, t)=v(L, t)=0, \int_{0}^{L} x u(x, t) d x=\int_{0}^{L} x v(x, t) d x=0,
\end{array}\right.
$$

where $L<\infty, T<\infty, \mu \in C^{1}((0, \alpha)), g_{1}(),. g_{2}():. \mathbb{R}^{+} \longrightarrow \mathbb{R}^{+}$ and $f_{1}(.,),. f_{2}(.,):. \mathbb{R}^{2} \longrightarrow \mathbb{R}$ are given functions, which will be specified later.

The problems related with localized frictional damping have extensively studied by many teams as [11], where the authors obtained an exponential rate of decay for the solution of the viscoelastic nonlinear wave equation:

$$
\begin{aligned}
u_{t t} & -\Delta u+f(x, t, u)+\int_{0}^{t} g_{1}(t-s) \Delta u(s) d s+a(x) u_{t} \\
& =0, \text { in }(0, L) \times(0, T),
\end{aligned}
$$

for damping term $a(x) u_{t}$ may be null for some part of the domain.

We used the techniques in [11]; we have proven in [8] the existence of a global solution using the potential well theory for the following viscoelastic system with nonlocal boundary condition and localized frictional damping

$$
\left\{\begin{array}{l}
u_{t t}-\frac{1}{x}\left(x u_{x}\right)_{x}+\int_{0}^{t} g_{1}(t-s) \frac{1}{x}\left(x u_{x}(x, s)\right)_{x} d s+a(x) u_{t}=|v|^{q+1}|u|^{p-1} u, \text { in }(0, L) \times(0, T), \\
v_{t t}-\frac{1}{x}\left(x v_{x}\right)_{x}+\int_{0}^{t} g_{2}(t-s) \frac{1}{x}\left(x v_{x}(x, s)\right)_{x} d s+a(x) v_{t}=|v|^{q+1}|u|^{p-1} v, \text { in }(0, L) \times(0, T), \\
u(x, 0)=u_{0}(x), u_{t}(x, 0)=u_{1}(x), x \in(0, \alpha), \\
v(x, 0)=v_{0}(x), v_{t}(x, 0)=v_{1}(x), x \in(0, \alpha), \\
u(\alpha, t)=v(\alpha, t)=0, \int_{0}^{\alpha} x u(x, t) d x=\int_{0}^{\alpha} x v(x, t) d x=0 .
\end{array}\right.
$$

Very recently, in ([9]), we study the following singular one-dimensional nonlinear equations that arise in generalized viscoelasticity with long-term memory:

$$
\left\{\begin{array}{l}
u_{t t}-\frac{1}{x}\left(x u_{x}\right)_{x}+\int_{0}^{t} g_{1}(t-s) \frac{1}{x}\left(x u_{x}(x, s)\right)_{x} d s=f_{1}(u, v), \text { in }(0, L) \times(0, T), \\
v_{t t}-\frac{1}{x}\left(x v_{x}\right)_{x}+\int_{0}^{t} g_{2}(t-s) \frac{1}{x}\left(x v_{x}(x, s)\right)_{x} d s=f_{2}(u, v), \text { in }(0, L) \times(0, T), \\
u(x, 0)=u_{0}(x), u_{t}(x, 0)=u_{1}(x), x \in(0, L), \\
v(x, 0)=v_{0}(x), v_{t}(x, 0)=v_{1}(x), x \in(0, L), \\
u(L, t)=v(L, t)=0, \int_{0}^{L} x u(x, t) d x=\int_{0}^{L} x v(x, t) d x=0 .
\end{array}\right.
$$


In view of the articles mentioned above in $([8,9,11])$, much less effort has been devoted to the existence of a global solution to the system of two singular nonlinear equations which arise in generalized viscoelasticity with localized frictional damping terms using the potential-well theory. Moreover, we prove a general decay result by constructing a Lyapunov functional and use it together with the perturbed energy method.

The structure of the work is as follows: To facilitate the description, firstly in Section 2, we give the fundamental definitions and theorems on function spaces that will be needed in the body of the paper and state the local existence theorem. In Section 3, the energy function $E(t)$ is defined and proved to be a nonincreasing function of time. Finally, the main result is obtained, which gives the general decay conditions:

$$
g_{i}^{\prime}(t) \leq-\xi(t) g_{i}^{r}(t), i=1,2 .
$$

\section{Preliminaries}

Let $L_{x}^{p}=L_{x}^{p}((0, L))$ be the weighted Banach space equipped with the norm

$$
\|u\|_{L_{x}^{p}}=\left(\int_{0}^{L} x|u|^{p} d x\right)^{1 / p}
$$

when $p=2$, we get a Hilbert space, and we denote by $H=L_{x}^{2}$, it provided with the finite norm

$$
\|u\|_{H}=\left(\int_{0}^{L} x u^{2} d x\right)^{1 / 2}
$$
norm

$V=V_{x}^{1}((0, L))$ be the Hilbert space equipped with the

$$
\|u\|_{V}=\left(\|u\|_{H}^{2}+\left\|u_{x}\right\|_{H}^{2}\right)^{1 / 2} .
$$

We get the following lemma by combining the Poincare inequality and (see [8]).

Lemma 1. Let $V_{0}$ space defined as follows

$$
V_{0}=\{u \in V \quad \text { such that } \quad u(L)=0\} .
$$

Then, for $2 \leq p 4$, we have

$$
\int_{0}^{L} x|v|^{p} d x \leq C_{*}\left\|v_{x}\right\|_{H=L_{x}^{2}(0, L)}^{p}, \forall u \in V_{0},
$$

where $C_{*}$ is a constant depending on $L$ and $p$ only and for $p=2, C_{*}=C_{p}$ is the Poincare constant.

Remark 2. It is clear that $\|u\|_{V 0}=\left\|u_{x}\right\|_{H}$ defines an equivalent norm on $V_{0}$.

The next theorem confirms that our problem has a local solution under some condition on $p$ and the relaxation func- tions $g_{i}$, the proof can be established by following the argument of [12].

Theorem 3. We take $\left(u_{0}, v_{0}\right) \in V_{0}^{2}$ and $\left(v_{1}, v_{2}\right) \in H^{2}$. If $p<3$ and

$$
g_{i}(0)>0,\left(1-\int_{0}^{\infty} g_{i}(s) d s\right)=l>0, \text { for } i=1,2,
$$

then, there exists $t *>0$ small enough such that the problem (1) has a unique local solution

$$
u \in C\left(0, t_{*} ; V_{0}\right) \cap C^{1}\left(0, t_{*} ; H\right) .
$$

Remark 4. The condition on $p$ is needed so that the embedding of $V_{0}$ in $L_{x}^{2}$ is Lipchitz.

We need the following assumptions to get our results.

$\left(\boldsymbol{G}_{1}\right)$ For $i=1,2, g_{i}(t): \mathbb{R}^{+} \longrightarrow \mathbb{R}^{+}$is a nonincreasing $C_{2}$ function such that

$$
\left\{\begin{array}{l}
g_{i}(s) \geq 0, g_{i}^{\prime}(s) \leq 0 \text { and, } \\
g_{i}(0) \geq 0,1-\int_{0}^{\infty} g_{i}(s) d s=l_{1}>0,
\end{array}\right.
$$

and

$\left(G_{2}\right)$

$$
g_{i}^{\prime}(t) \leq-\xi(t) g_{i}^{\sigma}(t), \quad t \geq 0,1 \leq \sigma<\frac{3}{2},
$$

where $\xi(t)$ is a positive differentiable function. It satisfies for some positive constant $l$

$$
\left|\frac{\xi^{\prime}(t)}{\xi(t)}\right| \leq l, \xi^{\prime}(t) \leq 0, \quad \int_{0}^{\infty} \xi(s) d s=+\infty, \forall t>0 .
$$

Furthermore, for any $t_{0}>0$ and $1<\sigma<3 / 2$, there exists a positive constant $C_{\sigma}$ such that

$$
\frac{t}{\left(1+\int_{t_{0}}^{t} \xi(s) d s\right)^{1 / 2(\sigma-1)}} \leq C_{\sigma}, \forall t \geq t_{0} .
$$

$\left(\boldsymbol{G}_{3}\right)$ We take

$$
\begin{aligned}
& f_{1}(u, v)=a|u+v|^{2(r+1)}(u+v)+b|u|^{r} u|v|^{r+2}, \\
& f_{2}(u, v)=a|u+v|^{2(r+1)}(u+v)+b|v|^{r} v|u|^{r+2},
\end{aligned}
$$

where $a, b>0$ are constants and $r>-1$.

One can easily verify that

$$
u f_{1}(u, v)+v f_{2}(u, v)=2(r+2) F(u, v), \forall(u, v) \in \mathbb{R}^{2},
$$


where

$$
F(u, v)=\frac{1}{2(r+2)}\left[a|u+v|^{2(r+2)}+2 b|u v|^{r+2}\right] .
$$

$\left(\boldsymbol{G}_{4}\right) \mu \geq 0, \mu>0$ in $\left(L_{0}, L\right]$, where $0 \leq L_{0} \leq L$.

Lemma 5. For $r>-1$, there exist $\eta>0$ such that for any $u, v$ $\in V \cap V_{0}(0, L)$, we have

$$
\|u+v\|_{L_{x}^{2(r+2)}}^{2(r+2)}+2\|u v\|_{L_{x}^{(r+2)}}^{(r+2)} \leq \eta\left(l_{1}\left\|u_{x}\right\|_{H}^{2}+l_{2}\left\|v_{x}\right\|_{H}^{2}\right)^{r+2} .
$$

Proof. From Minkowski inequality, we have

$$
\|u+v\|_{L_{x}^{2(r+2)}}^{2} \leq 2\left(\|u\|_{L_{x}^{2(r+2)}}^{2}+\|v\|_{L_{x}^{2(r+2)}}^{2}\right) .
$$

We apply successively Holder's and Young's inequalities we obtain

$$
\|u v\|_{L_{x}^{(r+2)}}^{(r+2)} \leq\|u\|_{L_{x}^{2(r+2)}}\|v\|_{L_{x}^{2(r+2)}} \leq c\left(l_{1}\left\|u_{x}\right\|_{H}^{2}+l_{2}\left\|v_{x}\right\|_{H}^{2}\right) .
$$

We combine the two previous inequalities and the embedding $V \cap V_{0}(0, L), \hookrightarrow L_{x}^{2(r+2)}(0, L)$, we get (20).

Lemma 6. There exist two positive constants $\Lambda_{1}$ and $\Lambda_{2}$ such that

$$
\begin{aligned}
& x\left|f_{i}(u, v)\right|^{2} d x \\
& \quad \leq \Lambda_{i}\left(l_{1} \int_{0}^{L} x u_{x}^{2} d x+l_{2} \int_{0}^{L} x v_{x}^{2} d x\right)^{2 r+3}, \forall x \in(0, L), i=1,2 .
\end{aligned}
$$

Proof. It is clear that

$$
\begin{aligned}
\left|f_{1}(u, v)\right| & \leq C\left(|u+v|^{2 r+3}+|u|^{r+1}|v|^{r+2}\right) \\
& \leq C\left[|u|^{2 r+3}|v|^{2 r+3}+|u|^{r+1}|v|^{r+2}\right],
\end{aligned}
$$

Applying Young's inequality with exponents $q=(2 r+3) /$ $(r+1), q^{\prime}=(2 r+3) /(r+2)$, in the last term in the above inequality, we get

$$
\left|f_{1}(u, v)\right| \leq C\left[|u|^{2 r+3}+|v|^{2 r+3}\right] .
$$

Consequently, by using Poincaré's inequality and (20), we obtain

$$
\begin{aligned}
\int_{0}^{L} x\left|f_{i}(u, v)\right|^{2} d x & \leq C\left(\left\|u_{x}\right\|_{H}^{2(2 r+3)}+\left\|v_{x}\right\|_{H}^{2(2 r+3)}\right) \\
& \leq \Lambda_{1}\left(l_{1}\left\|u_{x}\right\|_{H}^{2}+l_{2}\left\|u_{x}\right\|_{H}^{2}\right)^{(2 r+3)} .
\end{aligned}
$$

Similarly, we get the inequality for $f_{2}$. The proof is completed.
We define the energy function as

$$
\begin{aligned}
E(t)= & \frac{1}{2} \int_{0}^{L} x u_{t}^{2} d x+\frac{1}{2} \int_{0}^{L} x v_{t}^{2} d x+\frac{1}{2}\left(1-\int_{0}^{t} g_{1}(s) d s\right) \int_{0}^{L} x u_{x}^{2} d x \\
& +\frac{1}{2}\left(1-\int_{0}^{t} g_{1}(s) d s\right) \int_{0}^{L} x v_{x}^{2}(x, t) d x-\int_{0}^{L} F(u, v) d x \\
& +\frac{1}{2}\left(g_{1} \circ u_{x}\right)(t)+\frac{1}{2}\left(g_{2} \circ u_{x}\right)(t),
\end{aligned}
$$

where

$$
\left(g_{2} \circ u_{x}\right)(t)=\int_{0}^{L} \int_{0}^{t} x g(t-s)\left|u_{x}(x, t)-u_{x}(x, s)\right|^{2} d s d x .
$$

Lemma 7. Let $(u, v)$ be the solution of system (1) then for all $t \geq 0$

$$
\begin{aligned}
\frac{d}{d t}[E(t)]= & -\int_{0}^{L} x \mu(x) u_{t}^{2} d x-\int_{0}^{L} x \mu(x) v_{t}^{2} d x+\frac{1}{2}\left(g^{\prime} \circ u_{x}\right)(t) \\
& -\frac{1}{2} g_{1}(t) \int_{0}^{L} x u_{x}^{2}+\frac{1}{2}\left(g^{\prime} \circ v_{x}\right)(t)-\frac{1}{2} g_{2} \int_{0}^{L} x v_{x}^{2} d x .
\end{aligned}
$$

Hence, $E(t)$ is a nonincreasing function.

Proof. Multiplying the first and the second equations in (1) by $x u_{t}$ and $x v_{t}$, respectively, integrating over $(0, L)$, summing up, we obtain (30)

$$
\begin{aligned}
& \int_{0}^{L} x u_{t t} u_{t} d x-\int_{0}^{L}\left(x u_{x}\right)_{x} u_{t} d x+\int_{0}^{L} \int_{0}^{t} g_{1}(t-s)\left(x u_{x}(x, s)\right)_{x} d s u_{t} d x \\
& \quad+\int_{0}^{L} x v_{t t} v_{t} d x-\int_{0}^{L}(x v)_{x} v_{t}+\int_{0}^{L} \int_{0}^{t} g_{2}(t-s)\left(x v_{x}(x, s)\right)_{x} d s v_{t} d x \\
& =-\int_{0}^{L} x \mu(x) u_{t}^{2} d x-\int_{0}^{L} x \mu(x) v_{t}^{2} x \mu(x) v_{t}^{2} d x \\
& \quad+\int_{0}^{L}\left[a|u+v|^{2(r+1)}(u+v)+b|u|^{r} u|v|^{r+2}\right] x u_{t} d x \\
& \quad+\int_{0}^{L}\left[a|u+v|^{2(r+1)}(u+v)+b|v|^{r} v|u|^{r+2}\right] x v_{t} d x .
\end{aligned}
$$

By integration by parts, we obtain

$$
\begin{array}{r}
\int_{0}^{L} x u_{t t} u_{t} d x=\frac{1}{2} \frac{d}{d t}\left[\int_{0}^{L} x u_{t}^{2} d x\right], \\
\int_{0}^{L} x v_{t t} v_{t} d x=\frac{1}{2} \frac{d}{d t}\left[\int_{0}^{L} x v_{t}^{2} d x\right], \\
-\int_{0}^{L}\left(x u_{x}\right)_{x} u_{t} d x=\frac{1}{2} \frac{d}{d t}\left[\int_{0}^{L} x u_{x}^{2} d x\right],
\end{array}
$$




$$
\begin{aligned}
& -\int_{0}^{L}\left(x v_{x}\right)_{x} v_{t} d x=\frac{1}{2} \frac{d}{d t}\left[\int_{0}^{L} x v_{x}^{2} d x\right] \\
& \frac{1}{2(r+2)} \int_{0}^{L} x f_{1}(u, v) u u_{t} d x+\frac{1}{2(r+t)} \int_{0}^{L} x f_{2}(u, v) v v_{t} d x \\
& =\frac{1}{2(r+2)} \frac{d}{d t} \int_{0}^{L}\left[a|u+v|^{2(r+2)}+2 b|u v|^{r+2}\right] x d x,
\end{aligned}
$$

$$
\begin{aligned}
\int_{0}^{L} \int_{0}^{t} g_{1}(t-s)\left(x u_{x}(s)\right)_{x} d s u_{t}(t) d x \\
=\frac{1}{2} \frac{d}{d t}\left[\left(g_{1} \circ u_{x}\right)(t)-\int_{0}^{t} g_{1}(s) d s \int_{0}^{L} x u_{x}^{2} d x\right] \\
-\frac{1}{2}\left(g_{1}^{\prime} \circ u_{x}\right)(t)+\frac{1}{2} g_{1}(t) \int_{0}^{L} x u_{x}^{2} d x
\end{aligned}
$$

$$
\begin{aligned}
\int_{0}^{L} \int_{0}^{t} g_{2}(t-s)\left(x v_{x}(s)\right)_{x} d s v_{t}(t) d x \\
=\frac{1}{2} \frac{d}{d t}\left[\left(g_{2} \circ u_{x}\right)(t)-\int_{0}^{t} g_{2}(t) d s \int_{0}^{L} x v_{x}^{2} d x\right] \\
\quad-\frac{1}{2}\left(g_{2}^{\prime} \circ u_{x}\right)(t)+\frac{1}{2} g_{2}(t) \int_{0}^{L} x v_{x}^{2} d x
\end{aligned}
$$

Combining (32)-(2.22) in (31), we get (30).

\section{Global Existence}

In order to state and prove the global existence, we set the following notation

$$
\begin{aligned}
I(t):= & I(u(t), v(t))=\left(1-\int_{0}^{t} g_{1}(s) d s\right) \int_{0}^{L} x u_{x}^{2} d x \\
& +\left(1-\int_{0}^{t} g_{2}(s) d s\right) \int_{0}^{L} x v_{x}^{2} d x+\left(g_{1} \circ u_{x}\right)(t) \\
& +\left(g_{2} \circ v_{x}\right)(t)-2(r+2) \int_{0}^{L} x\left[a|u+v|^{2(r+2)}\right. \\
& \left.+2 b|u v|^{r+2}\right] d x, \\
J(t):= & J(u(t), v(t))=\frac{1}{2}\left(1-\int_{0}^{t} g_{1}(s) d s\right) \int_{0}^{L} x u_{x}^{2} d x \\
& +\frac{1}{2}\left(1-\int_{0}^{t} g_{2}(s) d s\right) \int_{0}^{L} x v_{x}^{2} d x+\frac{1}{2}\left(g_{1} \circ u_{x}\right)(t) \\
& +\frac{1}{2}\left(g_{2} \circ v_{x}\right)(t)-\int_{0}^{L} x\left[a|u+v|^{2(r+2)}+2 b|u v|^{r+2}\right] d x
\end{aligned}
$$

we remark that

$$
E(t)=J(t) \frac{1}{2} \int_{0}^{L} x u_{t}^{2} d x+\int_{0}^{L} x v_{t}^{2} d x
$$

Lemma 8. Assume that $\left(\boldsymbol{G}_{1}\right),\left(\boldsymbol{G}_{2}\right)$, and (20) hold also for any $\left(u_{0}, v_{0}\right) \in V_{0}^{2}$ and $\left(u_{1}, v_{1}\right) \in H_{2}$ satisfying

$$
I(0)>0, \beta:=\eta\left(\frac{2(r+2)}{(r+1)} E(0)\right)^{r+1}<1
$$

where

$$
E(0)=J(0)+\frac{1}{2} \int_{0}^{L} x u_{1}^{2} d x+\frac{1}{2} \int_{0}^{L} x v_{1}^{2} d x
$$

Then, there exists $t_{*}>0$ such that

$$
I(t)>0, \forall t \in\left[0, t_{*}\right) .
$$

Proof. Since $I(0)>0$, then from the continuity of $I(t)$, there exist $t_{m} \leq t_{*}$ such that $I(t) \geq 0$ for all $t \in\left[0, t_{m}\right)$; this implies that we have a maximum time value noting $T_{m}$ such that

$$
\left\{I\left(T_{m}\right)=0 \quad \text { and } \quad I(t)>0, \quad \text { for all } \quad 0 \leq t<T_{m}\right\} .
$$

From formulas of $J(t)$ and $I(t)$ together with $\left(\boldsymbol{G}_{1}\right)$, we have

$$
\begin{aligned}
J(t)= & \frac{r+1}{2(r+2)}\left[\left(1-\int_{0}^{t} g_{1}(s) d s\right) \int_{0}^{L} x u_{x}^{2} d s\right. \\
& \left.+\left(1-\int_{0}^{t} g_{2}(s) d s\right) \int_{0}^{L} x v_{x}^{2} d x\right] \\
& +\frac{r+1}{2(r+2)}\left[\left(g_{1} \circ u_{x}\right)(t)+\frac{1}{2}\left(g_{2} \circ v_{x}\right)(t)\right] \\
& +\frac{1}{2(r+2)} I(t) \geq \frac{r+1}{2(r+2)}\left[\left(l_{1} \int_{0}^{L} x u_{x}^{2} d x+l_{2} \int_{0}^{L} x v_{x}^{2} d x\right)\right. \\
& \left.+\left(g_{1} \circ u_{x}\right)(t)+\frac{1}{2}\left(g_{2} \circ v_{x}\right)(t)\right],
\end{aligned}
$$

hence,

$$
\begin{aligned}
l_{1} \int_{0}^{L} x u_{x}^{2} d x+l_{2} \int_{0}^{L} x v_{x}^{2} d x \\
\quad \leq \frac{2(r+2)}{r+1} J(t) \leq \frac{2(r+2)}{r+1} E(t) \\
\quad \leq \frac{2(r+2)}{r+1} E(0), \forall t \in\left[0, T_{m}\right),
\end{aligned}
$$


Recalling Lemma 5 and (41), we get

$$
\begin{aligned}
& 2(r+2) \int_{0}^{L} F\left(u\left(T_{m}\right), v\left(T_{m}\right)\right) d x \\
& \leq \eta\left(l_{1} \int_{0}^{L} x u_{x}^{2} d x+l_{2} \int_{0}^{L} x v_{x}^{2} d x\right)^{r+2} \\
& \leq \eta\left(\frac{2(r+2)}{r+1} E(0)\right)^{r+1}\left(l_{1} \int_{0}^{L} x u_{x}^{2} d x+l_{2} \int_{0}^{L} x v_{x}^{2} d x\right) \\
&= \beta\left(l_{1} \int_{0}^{L} x u_{x}^{2} d x+l_{2} \int_{0}^{L} x v_{x}^{2} d x\right) \\
&<\left(1-\int_{0}^{t} g_{1}(s) d s\right)\left(\int_{0}^{L} x u_{x}^{2} d x\right) \\
&+\left(1-\int_{0}^{t} g_{2}(s) d s\right)\left(\int_{0}^{L} x v_{x}^{2} d x\right) \\
&+\left(g_{1} \circ u_{x}\right)(t)+\left(g_{2} \circ v_{x}\right)(t),
\end{aligned}
$$

consequently

$$
\begin{aligned}
& \left(1-\int_{0}^{t} g_{1}(s) d s\right)\left(\int_{0}^{L} x u_{x}^{2} d x\right) \\
& +\left(1-\int_{0}^{t} g_{2}(s) d s\right)\left(\int_{0}^{L} x v_{x}^{2} d x\right)+\left(g_{1} \circ u_{x}\right)(t) \\
& +\left(g_{2} \circ v_{x}\right)(t)-2(r+2) \int_{0}^{L} x F(u, v) d x>0
\end{aligned}
$$

we deduce that $I(t)>0, \forall t \in\left[0, T_{m}\right)$. By repeating the procedure, $T_{m}$ is extended to $t_{*}$.

Theorem 9. Suppose that $\left(\boldsymbol{G}_{1}\right),\left(\mathbf{G}_{2}\right)$, and (20) hold. Then, for any $\left(u_{0}, v_{0}\right) \in V_{0}^{2}$ and $\left(u_{1}, v_{1}\right) \in H^{2}$ satisfying (41), the solution of system (1) is a bounded and globally in time.

Proof. To achieve the proof of this theorem, it suffices to show that $\left\|u_{x}\right\|_{H}^{2}+\left\|v_{x}\right\|_{H}^{2}+\left\|u_{t}\right\|_{H}^{2}+\left\|v_{t}\right\|_{H}^{2}$ is bounded independently of $t$. As $E(t)$ is a nonincreasing function, we have

$$
E(0) \geq E(t)
$$

in the other hand and for the definition of $I(t)$, we have

$$
\begin{aligned}
x[a \mid u & \left.+\left.v\right|^{2(r+2)}+2 b|u v|^{r+2}\right] d x \\
= & I(t)-\left(1-\int_{0}^{t} g_{1}(s) d s\right) \int_{0}^{L} x u_{x}^{2} d x \\
& -\left(1-\int_{0}^{t} g_{2}(s) d s\right) \int_{0}^{L} x v_{x}^{2} d x-\left(g_{1} \circ u_{x}\right)(t) \\
& -\left(g_{2} \circ v_{x}\right)(t),
\end{aligned}
$$

we introduce (49) into (50), we get

$$
\begin{aligned}
E(0) \geq & E(t)=\frac{1}{2} \int_{0}^{L} x u_{t}^{2} d x+\frac{1}{2} \int_{0}^{L} x v_{t}^{2} d x \\
+ & \frac{1}{2}\left(1-\int_{0}^{t} g_{1}(s) d s\right) \int_{0}^{L} x u_{x}^{2} d x \\
+ & \frac{1}{2}\left(1-\int_{0}^{t} g_{2}(s) d s\right) \int_{0}^{L} x v_{x}^{2}(x, t) d x \\
& +\frac{1}{2}\left(g_{1} \circ u_{x}\right)(t)+\frac{1}{2}\left(g_{2} \circ v_{x}\right)(t)+I(t),
\end{aligned}
$$

by using (14), (15), and (41), (51) yields

$$
\begin{aligned}
E(0) \geq & E(t) \geq \frac{1}{2} \int_{0}^{L} x u_{t}^{2} d x+\frac{1}{2} \int_{0}^{L} x v_{t}^{2} d x \\
& +\left(\frac{r+1}{2(r+2)}\right) l_{1} \int_{0}^{L} x u_{x}^{2} d x+\left(\frac{r+1}{2(r+2)}\right) l_{2} \int_{0}^{L} x v_{x}^{2} d x \\
\geq & \mu_{0}\left(\int_{0}^{L} x u_{t}^{2} d x+\int_{0}^{L} x v_{t}^{2} d x+\int^{L} x u_{x}^{2} d x+\int_{0}^{L} x v_{x}^{2} d x\right)
\end{aligned}
$$

So

$$
\left\|u_{x}\right\|_{H}^{2}+\left\|v_{x}\right\|_{H}^{2}+\left\|u_{t}\right\|_{H}^{2}+\left\|v_{t}\right\|_{H}^{2} \leq \tau E(0)
$$

where

$$
\tau:=\max \left\{2, \frac{2(r+2)}{(r+1) l_{1}}, \frac{2(r+2)}{(r+1) l_{2}}\right\} .
$$

The proof is completed.

\section{Decay of Solutions}

Throughout this section, we will study the asymptotic behavior of solutions' decay by constructing a suitable Lyapunov function; to do so, for $N, \varepsilon_{1}$, and $\varepsilon_{2}$ are positive constants, we define the following function as

$$
F(t):=E(t)+\varepsilon_{1} \Phi(t)+\varepsilon_{2} \chi(t)+\psi(t)
$$

where

$$
\begin{aligned}
\Phi(t):= & \xi(t) \int_{0}^{L} x u_{t} u d x+\xi(t) \int_{0}^{L} x v_{t} v d x \\
& +\frac{\xi(t)}{2} \int_{0}^{L} x \mu(x)\left(u^{2}+v^{2}\right) d x, \\
x(t):= & -\xi(t) \int_{0}^{L} x u_{t} \int_{0}^{t} g_{1}(t-s)(u(t)-u(s)) d s d x \\
& -\xi(t) \int_{0}^{L} x v_{t} \int_{0}^{t} g_{2}(t-s)(v(t)-v(s)) d s d x,
\end{aligned}
$$


and

$$
\psi(t):=\xi(t) \int_{0}^{L} x u_{t} h(x) u_{x} d x+\xi(t) \int_{0}^{L} x v_{t} h(x) v_{x} d x
$$

with $h \in C^{1}([0, L]), h(0)=h(L)=0,(x h(x))^{\prime} \leq x$,

In the first step, we prove the equivalence between $F(t)$ and $E(t)$ given in the following lemma.

Lemma 10. For a choice of $\varepsilon_{1}$ and $\varepsilon_{2}$ small enough, we find two positive constants $\alpha_{1}$ and $\alpha_{2}$ such that

$$
\alpha_{1} F(t) \leq E(t) \leq \alpha_{2} F(t) .
$$

Proof. By using Young inequality, follow by recalling Lemma 1 and the fact that $0<\xi(t) \leq \xi(0)$, we get

$$
\begin{aligned}
& \left|\varepsilon_{1} \xi(t) \int_{0}^{L} x u_{t} u d x\right| \leq \frac{\varepsilon_{1}}{2} \xi(0)\left(\int_{0}^{L} x u_{t}^{2} d x+C_{p} \int_{0}^{L} x u_{x}^{2} d x\right) \\
& \left|\varepsilon_{1} \xi(t) \int_{0}^{L} x v_{t} v d x\right| \leq \frac{\varepsilon_{1}}{2} \xi(0)\left(\int_{0}^{L} x v_{t}^{2} d x+C_{p} \int_{0}^{L} x v_{x}^{2} d x\right) \\
& \left|-\varepsilon_{2} \xi(t) \int_{0}^{L} x u_{t} \int_{0}^{t} g_{1}(t-s)(u(t)-u(s)) d s d x\right| \\
& \leq \frac{\varepsilon_{2}}{2} \xi(0)\left(\int_{0}^{L} x u_{t}^{2} d x+C_{p}\left(1-l_{1}\right)\left(g_{1} \circ u_{x}\right)(t)\right) \\
& \left|-\varepsilon_{2} \xi(t) \int_{0}^{L} x v_{t} \int_{0}^{t} g_{2}(t-s)(v(t)-v(s)) d s d x\right| \\
& \quad \leq \frac{\varepsilon_{2}}{2} \xi(0)\left(\int_{0}^{L} x v_{x}^{2} d x+C_{p}\left(1-l_{2}\right)\left(g_{2} \circ v_{x}\right)(t)\right) \\
& \quad \leq \frac{\xi(0)}{2}\|\mu\|_{\infty} C_{p}\left(\int_{0}^{L} x u_{x}^{2} d x+\int_{0}^{L} x v_{x}^{2} d x\right) \\
& \quad \leq \frac{\xi(0)}{2}\|h\| \infty\left(\int_{0}^{L} x v_{x}^{2} d x+\int_{0}^{L} x v_{t}^{2} d x\right) \\
& \quad \leq \frac{\xi(0)}{2}\|h\| \infty\left(\int_{0}^{L} x \mu(x) u_{x} d x \mid\right. \\
& \left.\mid \xi(t) \int_{0}^{L} x v_{t}^{2} h(x) v_{x} d x+\int_{0}^{L} x u_{t}^{2} d x\right)
\end{aligned}
$$

combining (60)-(66) in (55), we get

$$
\begin{aligned}
\mid F(t) & -N E(t) \mid \\
\leq & \left(\left(\frac{\varepsilon_{1}+\varepsilon_{2}}{2}\right) \xi(0)+\frac{\xi(0)}{2}\|h\|_{\infty}\right) \int_{0}^{L} x u_{t}^{2} d x \\
& +\left(\left(\frac{\varepsilon_{1}+\varepsilon_{2}}{2}\right) \xi(0)+\frac{\xi(0)}{2}\|h\|_{\infty}\right) \int_{0}^{L} x v_{t}^{2} d x \\
& +\left(\frac{1+\|\mu\|_{\infty}}{2} \varepsilon_{1} C_{p} \xi(0)+\frac{\xi(0)}{2}\|h\|_{\infty}(p+1)\right) \int_{0}^{L} x u_{x}^{2} d x \\
& +\left(\frac{1+\|\mu\|_{\infty}}{2} \varepsilon_{1} C_{p} \xi(0)+\frac{\xi(0)}{2}\|h\|_{\infty}(q+1)\right) \int_{0}^{L} x v_{x}^{2} d x \\
& +\frac{\varepsilon_{2}}{2} C_{p} \xi(0)\left(\left(1-l_{1}\right)\left(g_{1} \circ u_{x}\right)(t)+\left(1-l_{2}\right)\left(g_{2} \circ v_{x}\right)(t)\right) .
\end{aligned}
$$

If we choose $\varepsilon_{1}, \varepsilon_{2}$ small enough, and $N$ large enough we find $\alpha_{1}, \alpha_{2}>0$ such that (59) holds true.

Now, we state a Lemma corresponding to the boundness of $\left(\operatorname{gov}_{x}\right)(t)$. It will be used in the calculus.

Lemma 11. Let $w \in L^{\infty}((0, T) ; H)$ be such that $w_{x} \in L^{\infty}$ $((0, t) ; H)$ and $g$ be a continuous function on $[0, T]$ and suppose that. Then, there exists a constant $C>0$ such that

$$
\begin{aligned}
\left(g_{x} w_{x}\right)(t) \leq & C\left(\sup _{0<s<T}\|w(., s)\|_{H}^{2} \int_{0}^{t} g^{1-\theta}(s) d s\right)^{\rho-1 / \rho-1+\theta} \\
& \times\left(\int_{0}^{t} g^{\rho}(t-s)\left\|w_{x}(., s)\right\|_{H}^{2} d s\right)^{\theta / \rho-1+\theta} \\
& \forall 0<\theta<1 \text { and } \rho>1 .
\end{aligned}
$$

$$
\begin{aligned}
\left(\operatorname{gow}_{x}\right)(t) \leq & c\left(t\left\|w_{x}(., t)\right\|_{H}^{2}+\int_{0}^{t}\left\|w_{x}(., s)\right\|_{H}^{2} d s\right)^{\rho-1 / \rho} \\
\times & \left(\int_{0}^{t} g^{\rho}(t-s)\left\|w_{x}(., t)-w_{x}(., s)\right\|_{H}^{2} d s\right)^{1 / 2} \\
& \text { for all } \rho>1 .
\end{aligned}
$$

Proof.

(1) For any $\sigma>1$, we have

$$
\begin{aligned}
\left(g \circ w_{x}\right)(t)= & \int_{0}^{t}(g(t-s))^{(1-\theta) / \sigma}\left\|w_{x}(., t)-w_{x}(., s)\right\|^{2 / \sigma} \\
& \cdot(g(t-s))^{\sigma-1+\theta / \sigma}\left\|w_{x}(., t)-w_{x}(., s)\right\|^{2(\sigma-1) / \sigma} d s .
\end{aligned}
$$


Applying Holder's inequality with exponents $\sigma$ and $\sigma / \sigma$ -1 , we get

$$
\begin{aligned}
\left(g \circ w_{x}\right)(t) \leq & \left(\int_{0}^{t} g^{1-\theta}(t-s)\left\|w_{x}(., t)-w_{x}(., s)\right\|^{2} d s\right)^{1 / \sigma} \\
& \times\left(\int_{0}^{t} g^{\sigma-1+\theta / \sigma-1}(t-s)\left\|w_{x}(., t)-w_{x}(., s)\right\|^{2} d s\right)^{\sigma-1 / \sigma},
\end{aligned}
$$

We set $\sigma=(\rho-1+\theta) /(\rho-1)$, (71) yields

$$
\begin{aligned}
\left(g o w_{x}\right)(t) \leq & \left(\int_{0}^{t} g^{1-\theta}(t-s)\left\|w_{x}(., t)-w_{x}(., s)\right\|_{H}^{2} d s\right)^{\rho-1 / \rho-1+\theta} \\
& \times\left(\int_{0}^{t} g^{\rho}(t-s)\left\|w_{x}(., t)-w_{x}(., s)\right\|_{H}^{2}\right)^{\theta / \rho-1+\theta}
\end{aligned}
$$

It is easy to see that

$$
\begin{aligned}
& \int_{0}^{t} g^{1-\theta}(t-s)\left\|w_{x}(., t)-w_{x}(., s)\right\|_{H}^{2} d s \\
& \quad \leq C \sup _{0<s<T}\left\|w_{x}(., s)\right\|_{H}^{2} \int_{0}^{t} g^{1-\theta}(s) d s .
\end{aligned}
$$

We obtain (68) by combining (72) and (73).

(2) We set $\theta=1$ in (72) and it suffices to note that

$$
\begin{aligned}
& \int_{0}^{t}\left\|w_{x}(., t)-w_{x}(., s)\right\|_{H}^{2} d s \\
& \quad \leq 2 t\left\|w_{x}(., t)\right\|_{H}^{2}+2 \int_{0}^{t}\left\|w_{x}(., s)\right\|_{H}^{2} d s,
\end{aligned}
$$

to arrive at (69).

In the next, we present three lemmas in which we give an upper bound of each derivative's functions in $F(t)$.

Lemma 12. Suppose that $r>-1$ and (39) hold. Then

$$
\begin{aligned}
\Phi^{\prime}(t) \leq & \left(1+\frac{l}{2 \delta}\right) \xi(t) \int_{0}^{L} x u_{t}^{2} d x+\left(1+\frac{l}{2 \delta}\right) \xi(t) \int_{0}^{L} x v_{t}^{2} d x \\
& -\xi(t)\left(\frac{l_{1}-\delta C_{p} l}{2}-\frac{C_{p}\|\mu\|_{\infty}}{2}\right) \int_{0}^{L} x u_{x}^{2} d x \\
& -\xi(t)\left(\frac{l_{2}-\delta C_{p} l}{2}-\frac{C_{p}\|\mu\|_{\infty}}{2}\right) \int_{0}^{L} x v_{x}^{2} d x-\xi(t)
\end{aligned}
$$

$$
\begin{aligned}
& +\frac{\xi(t)}{2 l_{1}}\left(\int_{0}^{t} g_{1}^{2-\sigma}(s) d s\right)\left(g_{1}^{\sigma} \circ u_{x}\right)(t) \\
& +\frac{\xi(t)}{2 l_{2}}\left(\int_{0}^{t} g_{2}^{2-\sigma}(s) d s\right)\left(g_{2}^{\sigma} \circ v_{x}\right)(t) \\
& +\frac{\xi(t)}{2(r+2)}\left[a|u+v|^{2(r+2)}+2 b|u v|^{r+2}\right] d x .
\end{aligned}
$$

For any $\delta>0$.

Proof. After derivation of (56), we recall the differential equations in (1), we get

$$
\begin{aligned}
\Phi^{\prime}(t)= & \xi^{\prime}(t) \int_{0}^{L} x u_{t} u d x+\xi(t) \int_{0}^{L} x u_{t}^{2} d x+(t) \int_{0}^{L} x u_{t t} u d x \\
& \left.+\xi^{\prime}(t) \int_{0}^{L} x v_{t} v d x+\xi^{\prime} t\right) \int_{0}^{L} x v_{t}^{2} d x \\
& +\xi(t) \int_{0}^{L} x v_{t t} v d x+\frac{\xi^{\prime}(t)}{2} \int_{0}^{L} x \mu(x)\left(u^{2}+v^{2}\right) d x \\
& +\xi(t)\left(\int_{0}^{L} x \mu(x)\left(u_{t} u+v_{t} v\right) d x\right) \\
= & \xi^{\prime}(t) \int_{0}^{L} x u_{t} u d x+\xi(t) \int_{0}^{L} x u_{t}^{2} d x-\xi(t) \int_{0}^{L} x u_{x}^{2} d x \\
& +\xi(t) \int_{0}^{L} x u_{x} \int_{0}^{t} g_{1}(t-s) u_{x}(s) d s d x \\
& +\xi^{\prime}(t) \int_{0}^{L} x v_{t} v d x+\xi(t) \int_{0}^{L} x v_{t}^{2} d x-\xi(t) \int_{0}^{L} x v_{x}^{2} d x \\
& +\xi(t) \int_{0}^{L} x v_{x} \int_{0}^{t} g_{2}(t-s) v_{x}(s) d s d x \\
& +\frac{\xi(t)}{2(r+2)}\left[a|u+v|^{2(r+2)}+2 b|u v|^{r+2}\right] d x .
\end{aligned}
$$

By Young's inequality and from, $\left(\boldsymbol{G}_{1}\right),\left(\boldsymbol{G}_{2}\right)$, and Lemma 1 , we arrive at

$$
\begin{aligned}
\xi(t) \int_{0}^{L} x u_{x}(t)\left(\int_{0}^{t} g_{1}(t-s) u_{x}(s) d s\right) d x \\
\leq \frac{\xi(t)}{2} \int_{0}^{L} x u_{x}^{2} d x+\frac{\xi(t)}{2} \int_{0}^{L} x\left(\int_{0}^{t} g_{1}(t-s)\right. \\
\left.\cdot\left(\left|u_{x}(s)-u_{x}(t)\right|+\left|u_{x}(t)\right|\right) d s\right)^{2} d x \\
\leq \frac{\xi(t)}{2} \int_{0}^{L} x u_{x}^{2} d x+\frac{\xi(t)}{2}\left(1+\eta_{1}\right)\left(1+l_{1}\right)^{2} \int_{0}^{L} x u_{x}^{2}(t) d x \\
\quad+\frac{\xi(t)}{2}\left(1+\frac{1}{\eta_{1}}\right)\left(\int_{0}^{t} g_{1}^{2-\sigma}(s) d s\right)\left(g_{1}^{\sigma} \circ u_{x}\right)(t)
\end{aligned}
$$




$$
\begin{aligned}
= & \xi(t)\left(\frac{1+\left(1+\eta_{1}\right)\left(1-l_{1}\right)^{2}}{2}\right) \int_{0}^{L} x u_{x}^{2} d x \\
& +\frac{\xi(t)}{2}\left(1+\frac{1}{\eta_{1}}\right)\left(\int_{0}^{t} g_{1}^{2-\sigma}(s) d s\right)\left(g_{1}^{\sigma} \circ u_{x}\right)(t) \\
& +\frac{\xi(t)}{r+2} \int_{0}^{L}\left[a|u+v|^{2(r+2)}+2 b|u v|^{r+2}\right] d x,
\end{aligned}
$$

$$
\begin{aligned}
& \cdot\left(\int_{0}^{t} g_{1}^{2-\sigma}(s) d s\right)\left(g_{1}^{\sigma} \circ u_{x}\right)(t)+\frac{\xi(t)}{2}\left(1+\frac{1}{\eta_{2}}\right) \\
& \cdot\left(\int_{0}^{t} g_{2}^{2-\sigma}(s) d s\right)\left(g_{2}^{\sigma} \circ v_{x}\right)(t) \\
& +\frac{\xi(t)}{2(r+2)} \int_{0}^{L}\left[a|u+v|^{2(r+2)}+2 b|u v|^{r+2}\right] d x,
\end{aligned}
$$

similarly, we get

$$
\begin{aligned}
& \int_{0}^{L} x v_{x}(t)\left(\int_{0}^{t} g_{1}(t-s) v_{x}(s) d s\right) d x \\
& \leq \xi \xi(t)\left(\frac{1+\left(1+\eta_{2}\right)\left(1-l_{2}\right)^{2}}{2}\right) \int_{0}^{L} x v_{x}^{2} d x \\
& \quad+\frac{\xi(t)}{2}\left(1+\frac{1}{\eta_{2}}\right)\left(\int_{0}^{t} g_{2}^{2-\sigma}(s) d s\right)\left(g_{2}^{\sigma} \circ v_{x}\right)(t) .
\end{aligned}
$$

For any $\eta_{1}$ and $\eta_{2}>0$. We also have

$$
\begin{aligned}
\xi^{\prime}(t) \int_{0}^{L} x u_{t} u d x \leq & \frac{\xi(t)}{2}\left|\frac{\xi^{\prime}(t)}{\xi(t)}\right|\left(C_{p} \delta \int_{0}^{L} x u_{x}^{2} d x+\frac{1}{\delta} \int_{0}^{L} x u_{t}^{2} d x\right) \\
\leq & \frac{\xi(t)}{2}\left(C_{p} l \delta \int_{0}^{L} x u_{x}^{2} d x+\frac{l}{\delta} \int_{0}^{L} x u_{t}^{2} d x\right) \\
& \forall \delta>0
\end{aligned}
$$

and similarly, we get

$\xi^{\prime}(t) \int_{0}^{L} x v_{t} v d x \leq \frac{\xi(t)}{2}\left(C_{p} l \delta \int_{0}^{L} x v_{x}^{2} d x+\frac{l}{\delta} \int_{0}^{L} x v_{t}^{2} d x\right)$.

Also, by Lemma 1, we have

$$
\begin{aligned}
& \frac{\xi^{\prime}(t)}{2} \int_{0}^{L} x \mu(x)\left(u^{2}+v^{2}\right) d x \\
& \quad \leq\|\mu\|_{\infty} \frac{\xi(t)}{2}\left[C_{p}\left(\int_{0}^{L} x\left(u_{x}^{2}+v_{x}^{2}\right) d x\right] .\right.
\end{aligned}
$$

Combining (77)-(81) in (76) leads to

$$
\begin{aligned}
\Phi^{\prime}(t) \leq & \left(1+\frac{l}{2 \delta}\right) \xi(t) \int_{0}^{L} x u_{t}^{2} d x+\left(1+\frac{l}{2 \delta}\right) \xi(t) \int_{0}^{L} x v_{t}^{2} d x \\
& -\frac{\xi(t)}{2}\left[1-\left(1+\eta_{1}\right)\left(1-l_{1}\right)^{2}-\delta C_{p} l-C_{p}\|u\|_{\infty}\right] \\
& \cdot \int_{0}^{L} x u_{t}^{2} d x-\frac{\xi(t)}{2}\left[1-\left(1+\eta_{2}\right)\left(1-l_{2}\right)^{2}-\delta C_{p} l\right. \\
& \left.-C_{p}\|u\|_{\infty}\right] \int_{0}^{L} x u_{t}^{2} d x+\frac{\xi(t)}{2}\left(1+\frac{1}{\eta_{1}}\right)
\end{aligned}
$$

we choose $\eta_{1}=l_{1} / 1-l_{1}$ and $\eta_{2}=l_{2} / 1-l_{2}$, hence (75) is established.

Lemma 13. Suppose that $r>-1,\left(\boldsymbol{G}_{1}\right),\left(\boldsymbol{G}_{2}\right)$, and (41) hold. Then

$$
\begin{aligned}
\chi^{\prime}(t) \leq & \xi(t) \theta\left[2+c_{1}+c_{1}^{\prime}+2\left(1-l_{1}\right)^{2}\right]\left(\int_{0}^{L} x u_{x}^{2} d x\right) \\
& +\xi(t) \theta\left[2+c_{2}+c_{2}^{\prime}+2\left(1-l_{2}\right)^{2}\right]\left(\int_{0}^{L} x v_{x}^{2} d x\right) \\
& +\xi(t)\left[\theta-\left(\int_{0}^{t} g_{1}(s) d s\right)+\theta l\right]\left(\int_{0}^{L} x u_{t}^{2} d x\right) \\
& +\xi(t)\left[\theta-\left(\int_{0}^{t} g_{2}(s) d s\right)+\theta l\right]\left(\int_{0}^{L} x v_{t}^{2} d x\right) \\
& +\left[\frac{1}{2 \theta}+2 \theta+\frac{C_{p}(2+l)}{4 \theta}\right] \times \xi(t)\left(\int_{0}^{t} g_{1}^{2-\sigma}(s) d s\right) \\
& \cdot\left(g_{1}^{\sigma} \circ u_{x}\right)(t)+\left[\frac{1}{2 \theta}+2 \theta+\frac{C_{p}(2+l)}{4 \theta}\right] \\
& \times \xi(t)\left(\int_{0}^{t} g_{2}^{2-\sigma}(s) d s\right)\left(g_{2}^{\sigma} \circ v_{x}\right)(t) \\
& -\frac{C_{p}}{4 \theta} \xi(t) g_{1}(0)\left(g_{1}^{\prime} \circ u_{x}\right)(t) \\
& -\frac{C_{p}}{4 \theta} \xi(t) g_{2}(0)\left(g_{2}^{\prime} \circ v_{x}\right)(t),
\end{aligned}
$$

for any $\theta>0$.

Proof. A derivation of (57) gives

$$
\begin{aligned}
\chi^{\prime}(t)= & -\xi^{\prime}(t) \int_{0}^{L} x u_{t} \int_{0}^{t} g_{1}(t-s)(u(t)-u(s)) d s d x \\
& -\xi(t) \int_{0}^{L} x u_{t t} \int_{0}^{t} g_{1}(t-s)(u(t)-u(s)) d s d x \\
& -\xi(t) \int_{0}^{L} x u_{t} \frac{d}{d t}\left(\int_{0}^{t} g_{1}(t-s)(u(t)-u(s)) d s\right) d x \\
& -\xi^{\prime}(t) \int_{0}^{L} x v_{t} \int_{0}^{t} g_{2}(t-s)(v(t)-v(s)) d s d x \\
& -\xi(t) \int_{0}^{L} x v_{t t} \int_{0}^{t} g_{2}(t-s)(v(t)-v(s)) d s d x \\
& -\xi(t) \int_{0}^{L} x v_{t} \frac{d}{d t}\left(\int_{0}^{t} g_{2}(t-s)(v(t)-v(s)) d s\right) d x,
\end{aligned}
$$


by using Liebniz's formula, we get

$$
\begin{aligned}
\chi^{\prime}(t)= & -\xi^{\prime}(t) \int_{0}^{L} x u_{t} \int_{0}^{t} g_{1}(t-s)(u(t)-u(s)) d s d x \\
& -\xi(t) \int_{0}^{L} x u_{t t} \int_{0}^{t} g_{1}(t-s)(u(t)-u(s)) d s d x \\
& -\xi(t) \int_{0}^{L} x u_{t} \int_{0}^{t} g_{1}^{\prime}(t-s)(u(t)-u(s)) d s d x \\
& -\xi(t)\left(\int_{0}^{t} g_{1}(s) d s\right) \int_{0}^{L} x u_{t}^{2} d x \\
& -\xi^{\prime}(t) \int_{0}^{L} x v_{t} \int_{0}^{t} g_{2}(t-s)(v(t)-v(s)) d s d x \\
& -\xi(t) \int_{0}^{L} x v_{t t} \int_{0}^{t} g_{2}(t-s)(v(t)-v(s)) d s d x \\
& -\xi(t) \int_{0}^{L} x v_{t}^{t} \int_{0}^{t} g_{2}^{\prime}(t-s)(v(t)-v(s)) d s d x \\
& -\xi(t)\left(\int_{0}^{t} g_{2}(s) d s\right) \int_{0}^{L} x v_{t}^{2} d x .
\end{aligned}
$$

Recalling the differentials equation in (1), we get

$$
\begin{aligned}
\chi^{\prime}(t)= & -\xi^{\prime}(t) \int_{0}^{L} x u_{t}\left(\int_{0}^{t} g_{1}(t-s)(u(t)-u(s)) d s\right) d x \\
& +\xi(t) \int_{0}^{L} x \mu(x) u_{t}\left(\int_{0}^{t} g_{1}(t-s)(u(t)-u(s)) d s\right) d x \\
& +\xi(t) \int_{0}^{L} x u_{x}\left(\int_{0}^{t} g_{1}(t-s)\left(u_{x}(t)-u_{x}(s)\right) d s\right) d x \\
& -\xi(t) \int_{0}^{L} x\left(\int_{0}^{t} g_{1}(t-s) u_{x}(s) d s\right) \\
& +\left(\int_{0}^{t} g_{1}(t-s)\left(u_{x}(t)-u_{x}(s)\right) d s\right) d x \\
& -\xi(t) \int_{0}^{L}\left(x f_{1}(u, v)\right)\left(\int_{0}^{t} g_{1}(t-s)(u(t)-u(s)) d s\right) d x \\
& -\xi(t) \int_{0}^{L} x u_{t}\left(\int_{0}^{t} g_{1}^{\prime}(t-s)(u(t)-u(s)) d s\right) d x \\
& -\xi(t)\left(\int_{0}^{t} g_{1}(s) d s\right) \int_{0}^{L} x u_{t}^{2} d x \\
& -\xi^{\prime}(t) \int_{0}^{L} x v_{t}\left(\int_{0}^{t} g_{2}(t-s)(v(t)-v(s)) d s\right) d x \\
& +\xi(t) \int_{0}^{L} x \mu(x) v_{t}\left(\int_{0}^{t} g_{2}(t-s)(v(t)-v(s)) d s\right) d x \\
& +\xi(t) \int_{0}^{L} x v_{x}\left(\int_{0}^{t} g_{2}(t-s)\left(v_{x}(t)-v_{x}(s)\right) d s\right) d x \\
& -\xi(t) \int_{0}^{L} x\left(\int_{0}^{t} g_{2}(t-s)\left(v_{x}(s)\right) d s\right) \\
& +\left(\int_{0}^{t} g_{2}(t-s)\left(v_{x}(t)-v_{x}(s)\right) d s\right) d x
\end{aligned}
$$

$$
\begin{aligned}
& -\xi(t) \int_{0}^{L}\left(x f_{2}(u, v)\right)\left(\int_{0}^{t} g_{2}(t-s)(v(t)-v(s)) d s\right) d x \\
& -\xi(t) \int_{0}^{L} x v_{t}\left(\int_{0}^{t} g_{2}^{\prime}(t-s)(v(t)-v(s)) d s\right) d x \\
& -\xi(t)\left(\int_{0}^{t} g_{2}(s) d s\right) \int_{0}^{L} x v_{t}^{2} d x .
\end{aligned}
$$

We will estimate all term in (86) by Young's inequality, Lemma 1, $\left(\boldsymbol{G}_{1}\right)$, and $\left(\boldsymbol{G}_{2}\right)$.

$$
\begin{aligned}
& -\xi^{\prime}(t) \int_{0}^{L} x u_{t}\left(\int_{0}^{t} g_{1}(t-s)(u(t)-u(s)) d s\right) d x \\
& \leq \xi(t)\left|\frac{\xi^{\prime}(t)}{\xi(t)}\right|\left[\theta \int_{0}^{L} x u_{t}^{2} d x+\frac{C_{p}}{4 \theta}\left(\int_{0}^{t} g_{1}^{2-\sigma}(s) d s\right)\left(g_{1}^{\sigma} \circ u_{x}\right)(t)\right] \\
& \leq \theta l \xi(t) \int_{0}^{L} x u_{t}^{2} d x+\frac{C_{p}}{4 \theta} \xi(t)\left(\int_{0}^{t} g_{1}^{2-\sigma}(s) d s\right)\left(g_{1}^{\sigma} \circ u_{x}\right)(t), \\
& -\xi(t) \int_{0}^{L} x \mu(x) u_{t}\left(\int_{0}^{t} g_{1}(t-s)(u(t)-u(s)) d s\right) d x \\
& \leq \xi(t)\|\mu\|_{\infty}\left[\theta \int_{0}^{L} x u_{t}^{2} d x+\frac{C_{p}}{4 \theta}\left(\int_{0}^{t} g_{1}^{2-r}(s) d s\right)\left(g_{1}^{r} \circ u_{x}\right)(t)\right],
\end{aligned}
$$

and

$$
\begin{gathered}
-\xi(t) \int_{0}^{L} x u_{x}\left(\int_{0}^{t} g_{1}(t-s)\left(u_{x}(t)-u_{x}(s)\right) d s\right) d x \\
\leq \theta \xi(t) \int_{0}^{L} x u_{x}^{2} d x+\frac{1}{4 \theta} \xi(t)\left(\int_{0}^{t} g_{1}^{2-\sigma}(s) d s\right)\left(g_{1}^{\sigma} \circ u_{x}\right)(t), \\
-\xi(t) \int_{0}^{L} x\left(\int_{0}^{t} g_{1}(t-s) u_{x}(s) d s\right)\left(\int_{0}^{t} g_{1}(t-s)\left(u_{x}(t)-u_{x}(s)\right) d s\right) d x \\
\leq 2 \theta\left(1-l_{1}\right)^{2} \xi(t) \int_{0}^{L} x u_{x}^{2} d x+\left(2 \theta+\frac{1}{4 \theta}\right) \xi(t) \\
\cdot\left(\int_{0}^{t} g_{1}^{2-\sigma}(s) d s\right)\left(g_{1}^{\sigma} \circ u_{x}\right)(t), \\
-\xi(t) \int_{0}^{L} x u_{t}\left(\int_{0}^{t} g_{1}^{\prime}(t-s)(u(t)-u(s)) d s\right) d x \\
\leq \theta \xi(t) \int_{0}^{L} x u_{t}^{2} d x-\frac{g_{1}(0)}{4 \theta} C_{p} \xi(t)\left(g_{1}^{\prime} \circ u_{x}\right)(t),
\end{gathered}
$$

and

$$
\begin{aligned}
-\xi(t) & \int_{0}^{L} x f_{1}(u, v)\left(\int_{0}^{t} g_{1}(t-s)(u(t)-u(s)) d s\right) d x \\
\leq & \frac{C_{p}}{4 \theta} \xi(t)\left(\int_{0}^{t} g_{1}^{2-\sigma}(s) d s\right)\left(g_{1}^{\sigma} \circ u_{x}\right)(t) \\
& +c_{1} \theta \xi(t) \int_{0}^{L} x u_{x}^{2} d x+c_{2} \theta \xi(t) \int_{0}^{L} x v_{x}^{2} d x,
\end{aligned}
$$


where

$$
\left\{\begin{array}{l}
c_{1}:=\Lambda_{1}\left(\frac{2(r+2)}{(r+1)} E(0)\right)^{2(r+1)}, \\
c_{2}:=\Lambda_{2}\left(\frac{2(r+2)}{(r+1)} E(0)\right)^{2(r+1)}
\end{array}\right.
$$

By the same technique, we obtain estimations on integrals corresponding to $v, g_{2}$, and $f_{2}$

$$
\begin{gathered}
-\xi^{\prime}(t) \int_{0}^{L} x v_{t}\left(\int_{0}^{t} g_{2}(t-s)(v(t)-v(s)) d s\right) d x \\
\leq \theta l \xi(t) \int_{0}^{L} x v_{t}^{2} d x+\frac{C_{p} l}{4 \theta} \xi(t)\left(\int_{0}^{t} g_{2}^{2-\sigma}(s) d s\right)\left(g_{2}^{\sigma} \circ v_{x}\right)(t) \\
-\xi(t) \int_{0}^{L} x \mu(x) v_{t}\left(\int_{0}^{t} g_{2}(t-s)(v(t)-v(s)) d s\right) d x \\
\leq \xi(t)\|\mu\|_{\infty}\left[\theta \int_{0}^{L} x v_{t}^{2} d x+\frac{C_{p}}{4 \theta}\left(\int_{0}^{t} g_{2}^{2-\sigma}(s) d s\right)\left(g_{2}^{\sigma} \circ v_{x}\right)(t)\right] \\
\xi(t) \int_{0}^{L} x v_{x}\left(\int_{0}^{t} g_{2}(t-s)\left(v_{x}(t)-v_{x}(s)\right) d s\right) d x \\
\leq \theta \xi(t) \int_{0}^{L} x v_{x}^{2} d x+\frac{1}{4 \theta} \xi(t)\left(\int_{0}^{t} g_{2}^{2-\sigma}(s) d s\right)\left(g_{2}^{\sigma} \circ v_{x}\right)(t) \\
-\xi(t) \int_{0}^{L} x\left(\int_{0}^{t} g_{2}(t-s) v_{x}(s) d s\right) \\
\cdot\left(\int_{0}^{t} g_{2}(t-s)\left(v_{x}(t)-v_{x}(s)\right) d s\right) d x \\
\leq 2 \theta\left(1-l_{2}\right)^{2} \xi(t) \int_{0}^{L} x v_{x}^{2} d x+\left(2 \theta+\frac{1}{4 \theta}\right) \xi(t) \\
\cdot\left(\int_{0}^{t} g_{2}^{2-\sigma}(s) d s\right)\left(g_{2}^{\sigma} \circ u_{x}\right)(t) \\
-\xi(t) \int_{0}^{L} x v_{t}\left(\int_{0}^{t} g_{2}^{\prime}(t-s)(v(t)-v(s)) d s\right) d x \\
\leq \theta \xi(t) \int_{0}^{L} x v_{t}^{2} d x-\frac{g_{2}(0)}{4 \theta} C_{p} \xi(t)\left(g_{2}^{\prime} \circ v_{x}\right)(t)
\end{gathered}
$$

and

$$
\begin{aligned}
&- \frac{\xi(t)}{2(r+2)} \int_{0}^{L} x f_{2}(u, v)\left(\int_{0}^{t} g_{2}(t-s)(v(t)-v(s)) d s\right) d x \\
& \leq \frac{C_{p}}{4 \theta} \xi(t)\left(\int_{0}^{t} g_{2}^{2-\sigma}(s) d s\right)\left(g_{2}^{\sigma} \circ v_{x}\right)(t) \\
& \quad+c_{1}^{\prime} \theta \xi(t) \int_{0}^{L} x u_{x}^{2} d x+c_{2}^{\prime} \theta \xi(t) \int_{0}^{L} x v_{x}^{2} d x,
\end{aligned}
$$

where

$$
\left\{\begin{array}{l}
c_{1}^{\prime}:=\Lambda_{1}^{\prime}\left(\frac{2(r+2)}{(r+1)} E(0)\right)^{2(r+1)}, \\
c_{2}^{\prime}:=\Lambda_{2}^{\prime}\left(\frac{2(r+2)}{(r+1)} E(0)\right)^{2(r+1)} .
\end{array}\right.
$$

A combination of (87)-(93) into (86) yields (83).

Lemma 14. Suppose that $r>-1,\left(\boldsymbol{G}_{1}\right),\left(\boldsymbol{G}_{2}\right),\left(\boldsymbol{G}_{4}\right)$, and (41) hold. Then

$$
\begin{aligned}
\psi(t)^{\prime} \leq & \xi(t)\left[1+\|h\|_{\infty} \theta\left(2+\|\mu\|_{\infty}\right)\right]\left(\int_{0}^{L} x u_{x}^{2} d x\right) \\
& +\xi(t)\left[1+\|h\|_{\infty} \theta\left(2+\|\mu\|_{\infty}\right)\right]\left(\int_{0}^{L} x v_{x}^{2} d x\right) \\
& +\xi(t) \int_{0}^{L}\left[1+\|h\|_{\infty} \theta\left(\frac{1}{2 \theta}+\frac{1}{4 \theta} \mu(x)\right)\right] x u_{t}^{2} d x \\
& +\xi(t) \int_{0}^{L}\left[1+\|h\|_{\infty} \theta\left(\frac{1}{2 \theta}+\frac{1}{4 \theta} \mu(x)\right)\right] x v_{t}^{2} d x \\
& +\xi(t)\left[\|h\|_{\infty} \frac{C_{p}}{4 \theta}\right]\left(\int_{0}^{t} g_{1}^{2-\sigma}(s) d s\right)\left(g_{1}^{\sigma} \circ u_{x}\right)(t) \\
& +\xi(t)\left[\|h\|_{\infty} \frac{C_{p}}{4 \theta}\right]\left(\int_{0}^{t} g_{2}^{2-\sigma}(s) d s\right)\left(g_{2}^{\sigma} \circ v_{x}\right)(t) \\
& +\frac{\xi(t)}{2(r+2)} \int_{0}^{L} x\left[a|u+v|^{2(r+2)}+2 b|u v|^{r+2}\right] d x
\end{aligned}
$$

For any $\theta>0$.

Proof. We derivate (58) and integrate by part and we finish by using the differential equations in (1), we get

$$
\begin{aligned}
\psi^{\prime}(t)= & \xi^{\prime}(t) \int_{0}^{L} u_{t} x h(x) u_{x} d x+\xi^{\prime}(t) \int_{0}^{L} v_{t} x h(x) v_{x} d x \\
& +\xi(t) \int_{0}^{L} u_{t} x h(x) u_{t x} d x+(q+1) \xi^{\prime}(t) \int_{0}^{L} v_{t} x h(x) v_{t x} d x \\
& +\xi(t) \int_{0}^{L} u_{t t} x h(x) u_{x} d x+\xi(t) \int_{0}^{L} v_{t t} x h(x) v_{t x} d x \\
= & \xi^{\prime}(t) \int_{0}^{L} u_{t} x h(x) u_{x} d x+\xi^{\prime}(t) \int_{0}^{L} v_{t} x h(x) v_{x} d x \\
& -\frac{1}{2} \xi(t) \int_{0}^{L}(x h(x))^{\prime} u_{t}^{2} d x-\frac{1}{2} \xi(t) \int_{0}^{L}(x h(x))^{\prime} v_{t}^{2} d x \\
& -\frac{1}{2} \xi(t) \int_{0}^{L}(x h(x))^{\prime} u_{x}^{2} d x-\frac{1}{2} \xi(t) \int_{0}^{L}(x h(x))^{\prime} v_{x}^{2} d x \\
& -\xi(t) \int_{0}^{L} x \mu(x) h(x) u_{t} u_{x} d x-\xi(t) \int_{0}^{L} x \mu(x) h(x) v_{t} v_{x} d x
\end{aligned}
$$




$$
\begin{aligned}
& -\xi(t) \int_{0}^{L} x h(x) u_{x}\left(\int_{0}^{t} g_{1}(t-s)(u(t)-u(s)) d s\right) d x \\
& -\xi(t) \int_{0}^{L} x h(x) v_{x}\left(\int_{0}^{t} g_{2}(t-s)(v(t)-v(s)) d s\right) d x \\
& +\frac{\xi(t)}{2(r+2)} \int_{0}^{L}(x h(x))^{\prime}\left[a|u+v|^{2(r+2)}+2 b|u v|^{r+2}\right] d x,
\end{aligned}
$$

Applying Young's and Poincare's inequalities, we get

$$
\begin{aligned}
& \xi^{\prime}(t) \int_{0}^{L} u_{t} x h(x) u_{x} d x \\
& \leq \xi(t)\|h\|_{\infty}\left(\theta \int_{0}^{L} x u_{x}^{2} d x+\frac{1}{4 \theta} \int_{0}^{L} x u_{t}^{2} d x\right), \\
& \int_{0}^{L} x \mu(x) h(x) u_{t} u_{x} d x \\
& \leq\|h\|_{\infty}\left(\theta \int_{0}^{L} x \mu(x) u_{x}^{2} d x+\frac{1}{4 \theta} \int_{0}^{L} x \mu(x) u_{t}^{2} d x\right),
\end{aligned}
$$

finally

$$
\begin{aligned}
& \int_{0}^{L} x h(x) u_{x}\left(\int_{0}^{t} g_{1}(t-s)(u(t)-u(s)) d s\right) d x \\
& \quad \leq\|h\|_{\infty}\left(\theta \int_{0}^{L} x u_{x}^{2} d x+\frac{C_{p}}{4 \theta}\left(\int_{0}^{t} g_{1}^{2-r}(s) d s\right)\left(g_{1}^{r} \circ u_{x}\right)(t)\right)
\end{aligned}
$$

similarly

$$
\begin{gathered}
\xi^{\prime}(t) \int_{0}^{L} v_{t} x h(x) v_{x} d x \\
\leq \xi(t)\|h\|_{\infty}\left(\theta \int_{0}^{L} x v_{x}^{2} d x+\frac{1}{4 \theta} \int_{0}^{L} x v_{t}^{2} d x\right), \\
\int_{0}^{L} x \mu(x) h(x) v_{t} v_{x} d x \\
\leq\|h\|_{\infty}\left(\theta \int_{0}^{L} x \mu(x) v_{x}^{2} d x+\frac{1}{4 \theta} \int_{0}^{L} x \mu(x) v_{t}^{2} d x\right),
\end{gathered}
$$

and

$$
\begin{aligned}
& \int_{0}^{L} x h(x) v_{x}\left(\int_{0}^{t} g_{1}(t-s)(v(t)-v(s)) d s\right) d x \\
& \quad \leq\|h\|_{\infty}\left(\theta \int_{0}^{L} x v_{x}^{2} d x+\frac{C_{p}}{4 \theta}\left(\int_{0}^{t} g_{1}^{2-r}(s) d s\right)\left(g_{1}^{r} \circ v_{x}\right)(t)\right)
\end{aligned}
$$

Using (97)-(101) and $(x h(x))^{\prime} \leq x$, we obtain (95).

Next Theorem show that solutions decreases exponentially with respect to $\xi(t)$ and $\sigma$.
Theorem 15. Suppose that $r>-1,\left(\boldsymbol{G}_{1}\right)$, and $\left(\boldsymbol{G}_{2}\right)$ hold and taking $u_{0}, v_{0} \in V_{0}^{2}$, and $\left(u_{1}, v_{1}\right) \in H^{2}$ such that (41) hold true. Then, for each $t_{0}>0$, there exist positive constants $K$ and $k$ such that

$$
E(t) \leq \begin{cases}K e^{-k \int_{t_{0}}^{t} \xi(s) d s}, & \sigma=1, \\ K\left(1+\int_{t_{0}}^{t} \xi(s) d s\right)^{-1 / \sigma-1}, & 1<\sigma<\frac{3}{2}, \forall t \geq t_{0} .\end{cases}
$$

Proof. Since $g_{1}$ and $g_{2}$ is continuous and $g_{1}(0)>0, g_{2}(0)>0$ then for any $t_{0}>0$, we have

$$
\left\{\int_{0}^{t} g_{i}(s) d s \geq \int_{t_{0}}^{t} g_{i}(s) d s=g_{1,0}>0, \quad \forall t \geq t_{0}, i=1,2 .\right.
$$

As $\xi$ is a positive decreasing function hence $1<\xi(t) / \xi(0)$ and by recalling Lemmas $7,12,13,14$, and (101), we get

$$
\begin{aligned}
F^{\prime}(t)=E^{\prime}( & t)+\varepsilon_{1} \Phi^{\prime}(t)+\varepsilon_{2} \chi^{\prime}+\psi^{\prime}(t) \\
\leq-\int_{0}^{L} & {\left[\left(N-\frac{\|h\|_{\infty}}{4 \theta}\right) \mu(x)-\left(1+\frac{\|h\|_{\infty}}{4 \theta}\right)\right.} \\
& \left.-\varepsilon_{1}\left(1+\frac{1}{2 \delta}\right)+\varepsilon_{2}\left(g_{1,0}-\theta-\theta l\right)\right] \xi(t) x u_{t}^{2} d x \\
& -\int_{0}^{L}\left[\left(N-\frac{\|h\|_{\infty}}{4 \theta}\right) \mu(x)-\left(1+\frac{\|h\|_{\infty}}{4 \theta}\right)\right. \\
& \left.-\varepsilon_{1}\left(1+\frac{1}{2 \delta}\right)+\varepsilon_{2}\left(g_{2,0}-\theta-\theta l\right)\right] \xi(t) x v_{t}^{2} d x \\
& +\left(2 \varepsilon_{1}+1\right) \xi(t) \int_{0}^{L} x\left[a|u+v|^{2(r+2)}+2 b|u v|^{r+2}\right] d x \\
& +\left(\frac{1}{2}-\frac{\varepsilon_{2} \xi(0)}{4 \theta} C_{p} g_{1}(0)\right)\left(g_{1}^{\prime} \circ u_{x}\right)(t) \\
& +\left(\frac{1}{2}-\frac{\varepsilon_{2} \xi(0)}{4 \theta} C_{p} g_{2}(0)\right)\left(g_{2}^{\prime} \circ v_{x}\right)(t) \\
& -\left[\frac{\varepsilon_{1}}{2}\left(l_{2}-C_{p}\left(\delta l+\|\mu\|_{\infty}\right)\right)-\varepsilon_{2} \theta\left(1+c_{2}\right.\right. \\
& \left.\left.+c_{2}^{\prime}+2\left(1-l_{2}\right)^{2}\right)-\left(1+\|h\|_{\infty}\right) \theta\left(2+\|\mu\|_{\infty}\right)\right] \\
& \times \xi(t)\left(\int_{0}^{L} x v_{x}^{2} d x\right)+\left[\frac{\varepsilon_{1}}{2 l_{1}}\right. \\
& \left.+\varepsilon_{2}\left(\frac{1}{2 \theta}+2 \theta+\frac{2+l+C_{p}\|h\|_{\infty}+4 \theta}{4 \theta}\right)\right] \\
& \times \xi(t)\left(\int_{0}^{L} g_{1}^{2-\sigma}(s) d s\right)\left(g_{1}^{\sigma} \circ u_{x}\right)(t) \\
& +\left[\frac{\varepsilon_{1}}{2 l_{2}}+\varepsilon_{2}\left(\frac{1}{2 \theta}+2 \theta+\frac{2+l+C_{p}\|h\|_{\infty}+4 \theta}{4 \theta}\right)\right] \\
& \xi \xi(t)\left(\int_{0}^{L} g_{2}^{2-\sigma}(s) d s\right)\left(g_{2}^{\sigma} \circ u_{x}\right)(t) . \\
&
\end{aligned}
$$


We take $\delta<\left(1 / 2 C_{p} l\right) \min \left\{l_{1}, l_{2}\right\}$, hence

$$
\left\{\begin{array}{l}
\left(l_{1}-\delta C_{p} l\right)>\frac{l_{1}}{2} \\
\left(l_{2}-\delta C_{p} l\right)>\frac{l_{2}}{2}
\end{array}\right.
$$

Also, we take $\theta>\max \left\{g_{1,0} / 2(1+l), g_{2,0} / 2(1+l)\right\}$, hence

$$
\left\{\begin{array}{l}
\left(g_{1,0}-(1+l) \theta\right)<\frac{1}{2} g_{1,0} \\
\left(g_{2,0}-(1+l) \theta\right)<\frac{1}{2} g_{2,0} .
\end{array}\right.
$$

Now, we choose $\varepsilon_{2}$ small enough such

$$
\begin{aligned}
& k_{1}:=\left(\frac{1}{2}-\frac{\varepsilon_{2} \xi(0)}{4 \theta} C_{p} g_{1}(0)\right)>0, \\
& k_{1}:=\left(\frac{1}{2}-\frac{\varepsilon_{2} \xi(0)}{4 \theta} C_{p} g_{2}(0)\right)>0,
\end{aligned}
$$
that

As far as $\delta, \theta$, and $\varepsilon_{2}$ are fixed, we then pick $\varepsilon_{1}$ so small

$$
\begin{aligned}
& \left\{\begin{array}{l}
k_{3}:=\frac{\varepsilon_{1}}{4}\left(l_{1}-2 C_{p}\|\mu\|_{\infty}\right)-\varepsilon_{2} \theta\left(1+c_{1}+c_{1}^{\prime}+2\left(1-l_{1}\right)^{2}\right)-\left(1+\|h\|_{\infty} \theta\left(2+\|\mu\|_{\infty}\right)\right)>0, \\
k_{4}:=\frac{\varepsilon_{1}}{4}\left(l_{2}-2 C_{p}\|\mu\|_{\infty}\right)-\varepsilon_{2} \theta\left(1+c_{2}+c_{2}^{\prime}+2\left(1-l_{2}\right)^{2}\right)-\left(1+\|h\|_{\infty} \theta\left(2+\|\mu\|_{\infty}\right)\right)>0,
\end{array}\right. \\
& k_{5}:=\left[-\varepsilon_{1}\left(1+\frac{1}{2 \delta}\right)+\varepsilon_{2}\left(g_{1,0}-(1+l) \theta\right)\right]>0, \\
& k_{6}:=\left[-\varepsilon_{1}\left(1+\frac{1}{2 \delta}\right)+\varepsilon_{2}\left(g_{2,0}-(1+l) \theta\right)\right]>0, \\
& -\left\{\left[\frac{\varepsilon_{1}}{2 l_{1}}+\varepsilon_{2}\left(\frac{1}{2 \theta}+2 \theta+\frac{2+l+C_{p}\|h\|_{\infty}+4 \theta}{4 \theta}\right)\right]\left(\int_{0}^{t} g_{1}^{2-\sigma}(s) d s\right)\right\}>0,
\end{aligned}
$$

and

$$
\begin{aligned}
&-\{ {\left[\frac{\varepsilon_{1}}{2 l_{3}}+\varepsilon_{2}\left(\frac{1}{2 \theta}+2 \theta+\frac{2+l+C_{p}\|h\|_{\infty}+4 \theta}{4 \theta}\right)\right] } \\
&\left.\cdot\left(\int_{0}^{t} g_{2}^{2-\sigma}(s) d s\right)\right\}>0,
\end{aligned}
$$

Finally we choose $N>\|h\|_{\infty} / 4 \theta$ large enough such that

$$
\left(N-\frac{\|h\|_{\infty}}{4 \theta}\right) \mu(x)-\left(1+\frac{\|h\|_{\infty}}{4 \theta}\right)>0 .
$$

After fixed all this choices then from (14) and (27), we obtain for some $\sigma>0$,

$$
\begin{aligned}
F^{\prime}(t) \leq & -\sigma \xi(t)\left[\int_{0}^{L} x u_{t}^{2} d x+\int_{0}^{L} x v_{t}^{2} d x\right. \\
& -\int_{0}^{L} x\left[a|u+v|^{2(r+2)}+2 b|u v|^{r+2}\right] d x \\
& +\int_{0}^{L} x u_{x}^{2} d x+\int_{0}^{L} x v_{x}^{2} d x+\left(g_{1}^{\sigma} \circ u_{x}\right)(t) \\
& \left.+\left(g_{2}^{\sigma} \circ v_{x}\right)(t)\right] \leq-\gamma \xi(t) E(t), \forall t \geq t_{0} .
\end{aligned}
$$

We distingue two cases according $\sigma$
Case 16. $\sigma=1$

We recall lemma 10, and the estimate (111) gives

$$
F^{\prime}(t) \leq-\gamma \alpha_{1} \xi(t) F(t), \forall t \geq t_{0} .
$$

A simple integration of the above inequality over $\left(t_{0}, t\right)$ leads to

$$
F^{\prime}(t) \leq F\left(t_{0}\right) e^{\left(-\gamma \alpha_{1}\right) \int_{t_{0}}^{t} \xi(s) d s}, \forall t \geq t_{0}
$$

Hence, (102) is established.

Case 17. $1<\sigma<3 / 2$

By using (16), we get

$$
g_{i}(t)^{1-\sigma} \geq(\sigma-1)\left(\int_{t_{0}}^{t} \xi(s) d s\right)+g_{i}\left(t_{0}\right)^{1-\sigma}, \quad i=1,2 .
$$

So, for $\forall 0<\tau<2-\sigma<1$, (hence $(1-\tau / \sigma-1)>1$ ), we have

$$
\int_{0}^{\infty} g_{i}^{1-\tau}(s) d s \leq \int_{0}^{\infty} \frac{1}{\left[(\sigma-1)\left(\int_{t_{0}}^{t} \xi(s) d s\right)+g_{i}\left(t_{0}\right)^{1-\sigma}\right]^{1-\tau / \sigma-1}} d s
$$


In the other hand, by using the fact that $\int_{0}^{\infty} \xi(s) d s=+\infty$, we obtain

$$
\int_{0}^{\infty} g_{i}^{1-\tau}(s) d s<\infty, \forall 0<\tau<2-\sigma, \text { for } i=1,2
$$

So form (i) of Lemma 11 with $\theta=\tau$ and $\rho=\sigma$ and (41) yield

$$
\begin{aligned}
\left(g_{i} \circ w_{x}\right)(t) \leq & C_{i}\left(E(0) \int_{0}^{\infty} g_{i}^{1-\tau}(s) d s\right)^{\sigma-1 / \sigma-1+\tau} \\
& \cdot\left(\left(g_{i}^{\sigma} \circ w_{x}\right)(t)\right)^{\tau / \sigma-1+\tau} \\
\leq & C_{i}^{\prime}\left(\left(g_{i}^{\sigma} \circ w_{x}\right)(t)\right)^{\tau / \sigma-1+\tau},
\end{aligned}
$$

for $i=1,2$ and $w=u, v$, respectively, with $C_{i}^{\prime}$ are positive constants.

Therefore, for any $\sigma_{1}>1$, we arrive at

$$
\begin{aligned}
E^{\sigma_{1}}(t) \leq & C^{\prime \prime} E^{\sigma_{1}-1}(0)\left(\int_{0}^{L} x u_{t}^{2} d x+\int_{0}^{L} x v_{t}^{2} d x+\int_{0}^{L} x u_{x}^{2} d x\right. \\
& \left.+\int_{0}^{L} x v_{x}^{2} d x-\int_{0}^{L} x\left[a|u+v|^{2(r+2)}+2 b|u v|^{r+2}\right] d x\right) \\
& +C_{1}^{\prime \prime}\left(\left(g_{1} \circ u_{x}\right)(t)\right)^{\sigma_{1}}+C_{2}^{\prime \prime}\left(\left(g_{2} \circ v_{x}\right)(t)\right)^{\sigma_{1}} \\
\leq & C^{\prime \prime} E^{\sigma_{1}-1}(0)\left(\int_{0}^{L} x u_{t}^{2} d x+\int_{0}^{L} x v_{t}^{2} d x+\int_{0}^{L} x u_{x}^{2} d x\right. \\
& \left.+\int_{0}^{L} x v_{x}^{2} d x-\int_{0}^{L} x\left[a|u+v|^{2(r+2)}+2 b|u v|^{r+2}\right] d x\right) \\
& +C_{1}^{\prime \prime \prime}\left(\left(g_{1}^{\sigma} \circ u_{x}\right)(t)\right)^{\tau \sigma_{1} / \sigma-1+\tau} \\
& +C_{2}^{\prime \prime \prime}\left(\left(g_{2}^{\sigma} \circ v_{x}\right)(t)\right)^{\tau \sigma_{1} / \sigma-1+\tau} .
\end{aligned}
$$

By setting $\tau=1 / 2$ and $\sigma 1=2 \sigma-1$ which give ${ }^{\tau \sigma_{1} / \sigma-1+\tau=1}$, the estimate (119) becomes,

$$
\begin{aligned}
E^{\sigma_{1}}(t) \leq & \Gamma\left[\int_{0}^{L} x u_{t}^{2} d x+\int_{0}^{L} x v_{t}^{2} d x+\int_{0}^{L} x u_{x}^{2} d x+\int_{0}^{L} x v_{x}^{2} d x\right] \\
& -\int_{0}^{L} x\left[a|u+v|^{2(r+2)}+2 b|u v|^{r+2} d x\right. \\
& \left.+\left(g_{1}^{\sigma} \circ u_{x}\right)(t)+\left(g_{2}^{\sigma} \circ v_{x}\right)(t)\right],
\end{aligned}
$$

for some $\Gamma>0$. Form the equivalence between $F$ and $E$ and by combining the above inequality with (111), we obtain

$$
F^{\prime}(t) \leq-\frac{\sigma}{\Gamma} \xi(t) E^{\sigma_{1}}(t) \leq-\frac{\sigma}{\Gamma} \alpha_{1}^{\sigma_{1}} F^{\sigma_{1}}(t), \quad \forall t \geq t_{0} .
$$

By twice integration over $\left(t_{0}, t\right)$ and $\left(t_{0}, \infty\right)$ of (119) successively leads to

$$
F(t) \leq C_{1}^{*}\left(1+\int_{t_{0}}^{t} \xi(s) d s\right)^{-1 / \sigma_{1}-1}, \quad \forall t \geq t_{0},
$$

$\int_{t_{0}}^{\infty} F(t) d t \leq C_{1}^{*} \int_{t_{0}}^{\infty} \frac{1}{\left(1+\int_{t_{0}}^{t} \xi(s) d s\right)^{1 / \sigma_{1}-1}} d t$.

Since ${ }^{1 / \sigma_{1}-1>0}$ and $\left(1+\int_{t_{0}}^{t} \xi(s) d s\right) \longrightarrow+\infty$ as $t \longrightarrow+\infty$, we get

$$
\int_{t_{0}}^{\infty} F(t) d t \leq \infty
$$

Again from (121), we have

$$
t F(t) \leq \frac{C_{1}^{*} t}{\left(1+\int_{t_{0}}^{t} \xi(s) d s\right)^{1 / \sigma_{1}-1}} \leq C_{\sigma}, \quad \forall t \geq t_{0},
$$

which implies that

$$
\sup _{t \geq t_{0}} t F(t)<\infty \text {. }
$$

Summing (123) and (125), we get

$$
\int_{t_{0}}^{\infty} F(t) d t+\sup _{t \geq t_{0}}(t F(t))<\infty
$$

By recalling (ii) of Lemma 11 with $\rho=\sigma$, Lemma 10 , and by using (27) and the above bounded, we have

$$
\begin{aligned}
\left(g_{i} \circ w_{x}\right)(t) \leq & C_{i 2}^{*}\left(t\left\|w_{x}(x, t)\right\|_{H}^{2}+\int_{0}^{t}\left\|w_{x}(x, s)\right\|_{H}^{2} d s\right)^{\sigma-1 / \sigma} \\
& \times\left(\int_{0}^{t} g_{i}^{\sigma}(t-s)\left\|w_{x}(x, t)-w_{x}(x, s)\right\|_{H}^{2} d s\right)^{1 / \sigma} \\
\leq & C_{i 2}^{*}\left(t F(t)+\int_{t_{0}}^{t} F(s) d s\right)^{\sigma-1 / \sigma}\left(\left(g_{i}^{\sigma} \circ w_{x}\right)(t)\right)^{1 / \sigma} \\
\leq & C_{i 3}^{*}\left(\left(g_{i}^{\sigma} \circ w_{x}\right)(t)\right)^{1 / \sigma},
\end{aligned}
$$

Hence,

$$
\left(g_{i}^{\sigma} \circ w_{x}\right)(t) \geq\left(C_{i 3}^{*}\right)^{-\sigma}\left(\left(g_{i} \circ w_{x}\right)(t)\right)^{\sigma},
$$

for $i=1,2, w=u, v$, respectively, and some positive constants $C_{i 3}^{*}$. 

find

Consequently, introduce (128) in (111) and in, (118), we

$$
\begin{aligned}
F^{\prime}(t) \leq & -C_{4} \xi(t)\left[\int_{0}^{L} x u_{t}^{2} d x+\int_{0}^{L} x v_{t}^{2} d x+\int_{0}^{L} x u_{x}^{2} d x\right. \\
& +\int_{0}^{L} x v_{x}^{2} d x-\int_{0}^{L} x\left[a|u+v|^{2(r+2)}+2 b|u v|^{r+2}\right] d x \\
& \left.+\left(\left(g_{1} \circ u_{x}\right)(t)\right)^{\sigma}+\left(\left(g_{2} \circ v_{x}\right)(t)\right)^{\sigma}\right]
\end{aligned}
$$

and

$$
\begin{aligned}
E^{\sigma}(t) \leq & C_{5} \xi(t)\left[\int_{0}^{L} x u_{t}^{2} d x+\int_{0}^{L} x v_{t}^{2} d x+\int_{0}^{L} x u_{x}^{2} d x+\int_{0}^{L} x v_{x}^{2} d x\right. \\
& -\int_{0}^{L} x\left[a|u+v|^{2(r+2)}+2 b|u v|^{r+2}\right] d x+\left(\left(g_{1} \circ u_{x}\right)(t)\right)^{\sigma} \\
& \left.+\left(\left(g_{2} \circ v_{x}\right)(t)\right)^{\sigma}\right]
\end{aligned}
$$

for all $t \geq 0$ and some positive constant $C_{4}, C_{5}$.

By combining the last two inequalities and along the equivalence between $F$ and $E$, we obtain

$$
F^{\prime}(t) \leq-C_{6} \xi(t) F^{\sigma}(t), \quad \forall t \geq t_{0}
$$

for some constant $C_{6}>0$.

A simple integration of (131) over $\left(t_{0}, t\right)$ gives

$$
F(t) \leq C_{9}\left(1+\int_{t_{0}}^{t} \xi(s) d s\right)^{-1 / \sigma-1}, \quad \forall t \geq t_{0} .
$$

The proof is completed by using (59).

\section{Data Availability}

No data were used to support this study.

\section{Conflicts of Interest}

The authors declare that there is no conflict of interests regarding the publication of this manuscript. The authors declare that they have no competing interests.

\section{Authors' Contributions}

The authors contributed equally in this article. They have all read and approved the final manuscript.

\section{References}

[1] B. Cahlon, D. M. Kulkarni, and P. Shi, "Stepwise stability for the heat equation with a nonlocal constraint," SIAM Journal on Numerical Analysis, vol. 32, no. 2, pp. 571-593, 1995.
[2] Y. S. Choi and K. Y. Chan, "A parabolic equation with nonlocal boundary conditions arising from electrochemistry," Nonlinear Analysis: Theory, Methods \& Applications, vol. 18, no. 4, pp. 317-331, 1992.

[3] A. V. Kartynnik, "Three-point boundary value problem with an integral space-variable condition for a second order parabolic equation," Differential Equations, vol. 26, pp. 11601162, 1990.

[4] L. S. Pulkina, "A nonlocal problem with integral conditions for hyperbolic equations," Electronic Journal of Differential Equations, vol. 45, pp. 1-6, 1999.

[5] L. S. Pulkina, "TheL 2 solvability of a nonlocal problem with integral conditions for a hyperbolic equation," Differential Equations, vol. 36, no. 2, pp. 316-318, 2000.

[6] N. I. Yurchuk, "Mixed problem with an integral condition for certain parabolic equations," Differential Equations, vol. 22, pp. 1457-1463, 1986.

[7] P. Shi and M. Shillor, "On design of contact patterns in one dimensional thermoelasticity," in Theoretical aspects of industrial design, SIAM, Philadelphia, 1992.

[8] S. Boulaaras and N. Mezouar, "Global existence and decay of solutions of a singular nonlocal viscoelastic system with a nonlinear source term, nonlocal boundary condition, and localized damping term," Mathematical Methods in the Applied Sciences, vol. 43, no. 10, pp. 6140-6164, 2020.

[9] S. Boulaaras, R. Guefaifia, and N. Mezouar, "Global existence and decay for a system of two singular one-dimensional nonlinear viscoelastic equations with general source terms," Applicable Analysis, pp. 1-25, 2020.

[10] W. Liu, Y. Sun, and G. Li, "On decay and blow-up of solutions for a singular nonlocal viscoelastic problem with a nonlinear source term. Topol," Methods Nonlinear Analysis, vol. 49, pp. 299-323, 2017.

[11] M. M. Cavalcanti, N. Valeria, D. Cavalcanti, and J. A. Soriano, "Exponential decay for the solution of semilinear viscoelastic wave equations with localized damping," Electronic Journal of Differential Equations, vol. 2002, no. 44, pp. 1-14, 2002.

[12] P. Shi, "Weak solution to an evolution problem with a nonlocal constraint," SIAM Journal on Mathematical Analysis, vol. 24, no. 1, pp. 46-58, 1993. 